\title{
Hydrostatic Compression Effects on Fifth-Group Element Superconductors V, Nb, and Ta Subjected to High-Pressure Torsion
}

\author{
Masaki Mito ${ }^{1, *}$, Shun Shigeoka ${ }^{1}$, Hirotaka Kondo ${ }^{1}$, Nozomi Noumi ${ }^{1}$, Yuichiro Kitamura ${ }^{1}$, Kunihiko Irie ${ }^{1}$, \\ Kazuma Nakamura ${ }^{1}$, Seishi Takagi ${ }^{1}$, Hiroyuki Deguchi ${ }^{1}$, Takayuki Tajiri ${ }^{2}$, Mamoru Ishizuka ${ }^{3}$, \\ Terukazu Nishizaki ${ }^{4}$, Kaveh Edalati ${ }^{5,6}$ and Zenji Horita ${ }^{5,6}$ \\ ${ }^{1}$ Graduate School of Engineering, Kyushu Institute of Technology, Kitakyushu 804-8550, Japan \\ ${ }^{2}$ Faculty of Science, Fukuoka University, Fukuoka 814-0180, Japan \\ ${ }^{3}$ Center for Scientific Instrument Renovation and Manufacturing Support, Osaka University, Toyonaka 560-8531, Japan \\ ${ }^{4}$ Department of Electrical Engineering, Kyushu Sangyo University, Fukuoka 813-8503, Japan \\ ${ }^{5}$ Department of Materials Science and Engineering, Faculty of Engineering, Kyushu University, Fukuoka 819-0395, Japan \\ ${ }^{6}$ International Institute for Carbon-Neutral Energy Research (WPI-I2CNER), Kyushu University, Fukuoka 819-0395, Japan
}

In fifth-group element superconductors $\mathrm{V}, \mathrm{Nb}$, and $\mathrm{Ta}$, the increase in superconducting transition temperature $\left(T_{\mathrm{c}}\right)$ was attempted by using both high-pressure torsion (HPT) and additional hydrostatic pressure (HP) compression. The former brings about the grain refinement and strain accumulation in the unit-cell level. The additional compression for severely strained superconductors triggers strengthening intergrain-contact and/or structural deformation in the unit-cell level. The manner of the appearance of the above two effects depends on the kind of elements: First, in V, there is no prominent effect of HPT, comparing to the hydrostatic compression effects on its non-strained material. Next, in Ta, the effect of strengthening intergrain-contact appears at small hydrostatic compression, resulting in temporal increase in $T_{\mathrm{c}}$. Finally, $\mathrm{Nb}$ exhibits prominent increase in $T_{\mathrm{c}}$ by both effects and, in particular, the structural deformation in the unit-cell level promotes the increase in $T_{\mathrm{c}}$. Thus, the accumulation of residual strain in the level of starting material can be a promising work to manipulate $T_{\mathrm{c}}$ under HP compression. [doi:10.2320/matertrans.MF201932]

(Received February 25, 2019; Accepted May 7, 2019; Published June 28, 2019)

Keywords: high-pressure torsion, hydrostatic pressure, superconductors, structural deformation, intergrain-contact, structural deformation

\section{Introduction}

Bulk superconductivity exhibits macroscopic phase coherence in the Josephson arrays composed of superconducting grains. In late 1950's-early1970's, the superconductivity property at zero magnetic field, ex. superconducting transition temperature $T_{\mathrm{c}}$, had been found to depend on both the sample purity and residual strain in single element superconductors such as $\mathrm{Re},{ }^{1)} \mathrm{Ta},{ }^{2)}$ and $\mathrm{V}^{3)}$ Generally, the shear strain reduces crystallinity, and it is usually not favorable for macroscopic phase coherence. However, it had been known that it rarely works toward favorable manner in $\mathrm{Re},{ }^{1)}$ thin film and amorphus $\mathrm{Al},{ }^{4)}$ and so on. Furthermore when considering the fine-grain superconductors, it has been experimentally confirmed that $T_{\mathrm{c}}$ can increase with strengthening the intergrain coupling., ${ }^{5,6}$ This effect is theoretically expected to be enhanced in a system with smaller grain size. ${ }^{7)}$

Recently by using severe plastic deformation (SPD), ${ }^{8,9)}$ the improvement of functionality in superconductors is successfully conducted. ${ }^{10-12)}$ The SPD process plays the role of accumulating dislocations, resulting in the grain refinement at the submicrometer or nanometer ranges as shown in Fig. 1. It is known to be effective in increasing $T_{\mathrm{c}}$ of superconductors such as $\mathrm{Nb}^{10)}$ and Re. ${ }^{12)}$ There we have to consider both the intergrain network via grain boundary and intragrain crystallinity as factors to influence $T_{\mathrm{c}}$. The strain installed in the SPD process is relatively evaluated via semi-experimental manner: In the case of high-pressure torsion (HPT), ${ }^{13-17)}$ which is one of the SPD techniques along with equal-channel

*Corresponding author, E-mail: mitoh@mns.kyutech.ac.jp

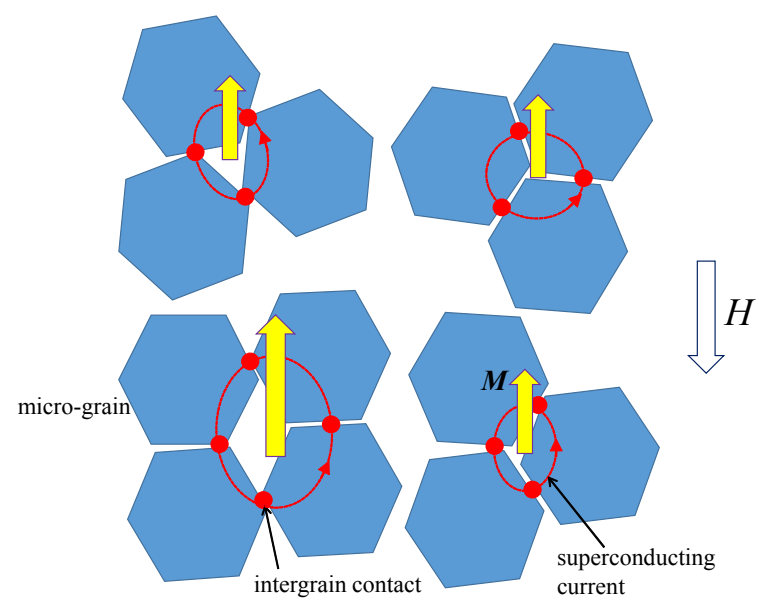

Fig. 1 Intergrain network consisting of superconducting grains. Grains and intergrain-contacts are presented with hexagons and red closed circles, respectively. The diamagnetic moments by the surface current displayed with red dotted curves are presented with yellow arrows. In the magnetic measurements, the summation of yellow $M$ vectors against the magnetic field $H$ is observed.

angular pressing ${ }^{18)}$ and accumulative roll bonding, ${ }^{19,20)}$ the revolution number $N$ is a parameter to pursue the grain refinement. In order to elucidate both effects on $T_{\mathrm{c}}$, we compress three fifth-element superconductors such as $\mathrm{V}, \mathrm{Nb}$, and Ta subjected to HPT as illustrated in Fig. 2(a).

In the periodic table containing 118 elements, there are 29 superconductors at ambient pressure, and 11 elements of them exhibit the increase in $T_{\mathrm{c}}$ under hydrostatic pressure (HP). ${ }^{21,22)}$ Three fifth-element superconductors such as V, $\mathrm{Nb}$, and $\mathrm{Ta}$ has nominal $T_{\mathrm{c}}$ of $5.3 \mathrm{~K}, 9.2 \mathrm{~K}$, and $4.5 \mathrm{~K}$, 
(a) High-pressure torsion (HPT) -- controlling initial condition --

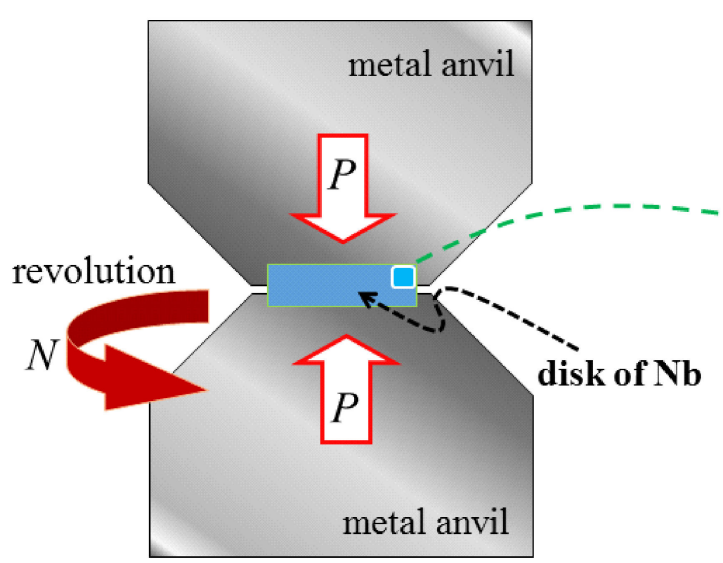

Change in Structure

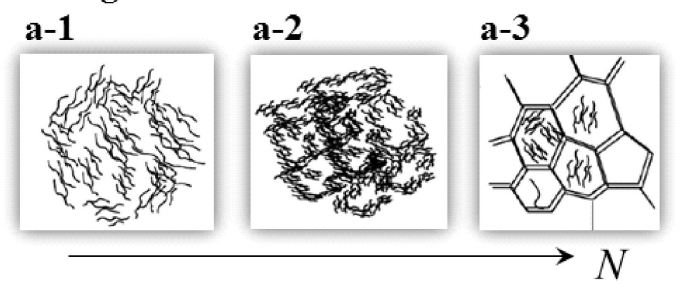

(b) Hydrostatic pressure experiment using diamond anvil cell (DAC)
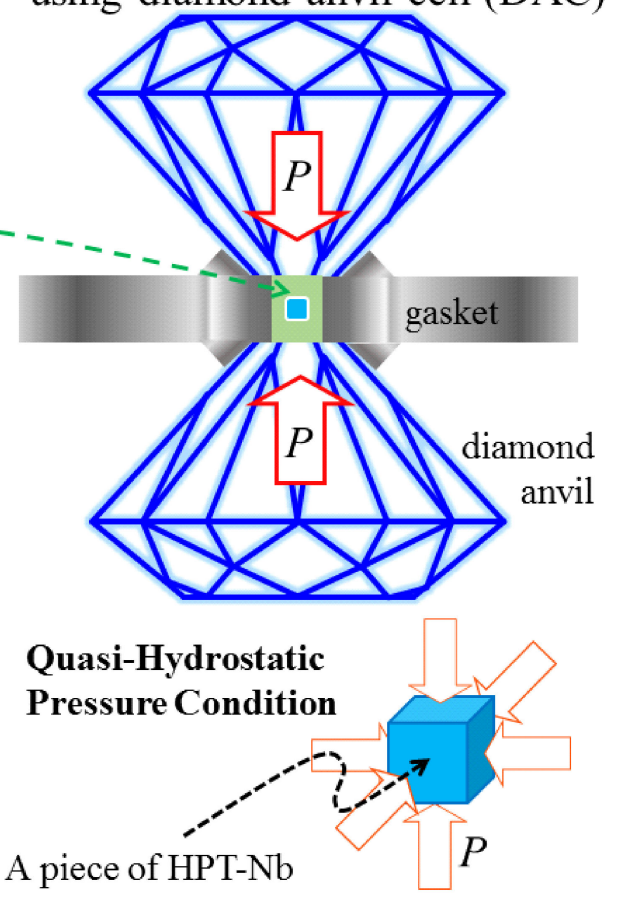

Fig. 2 Strategy of the present study in the case of $\mathrm{Nb}^{33}$ ) (a) In high-pressure torsion (HPT) for controlling the initial condition, pressurization and revolution are conducted by using two metal anvils. As the revolution number $(N)$ increases, dislocations are accumulated (a-1), and the grain size largely changes. The small-angle grain boundaries gradually transform to large-angle grain boundaries with increasing $N((\mathrm{a}-2) \rightarrow(\mathrm{a}-3))$. Finally due to the balance between generation and annihilation of dislocations, the grain size remains unchanged despite the increase in $N$. (b) A few pieces of sample cut from the HPT-processed V, Nb, and Ta are placed in diamond anvil cell (DAC) to conduct the hydrostatic pressure experiment. There, the specimen is pressed in a quasi-hydrostatic pressure condition using the DAC.

Table 1 Previous studies on hydrostatic pressure (HP) experiments for V. PTM: pressure-transmitting medium; nP-iA: 1:1 mixture of npentane and isoamyl alcohol, and Ap-J: Apiezon-J oil. CY: cylindrical sample, AR: as-received sample, S: single-crystal, SP: small pieces, ARC: arc-melted, and HPT: high-pressure torsion. $N$ denotes the revolution number of the HPT procedure performed at 6 GPa.

\begin{tabular}{|c|c|c|c|c|c|c|c|c|}
\hline year & authors & sample & $\begin{array}{l}\max . P \\
{[\mathrm{GPa}]}\end{array}$ & $\begin{array}{c}T_{\mathrm{c}}(P=0) \\
{[\mathrm{K}]}\end{array}$ & $\begin{array}{l}\mathrm{d} T_{\mathrm{c}} / \mathrm{d} P \\
{[\mathrm{~K} / \mathrm{GPa}]}\end{array}$ & $\begin{array}{c}\max . T_{\mathrm{C}} \\
{[\mathrm{K}]}\end{array}$ & PTM & ref \\
\hline 1966 & Gardner and Smith & $\mathrm{CY}$ of $\mathrm{AR}, \mathrm{ARC}$ & ca. 1 & $4.92-5.05,5.20$ & +0.11 & 5.31 & - & {$[23]$} \\
\hline 1968 & Köhnlein & - & - & - & +0.176 & - & - & {$[24]$} \\
\hline 1972 & Smith & $\mathrm{CY}$ after ARC & 2.0 & 5.272 & +0.065 & 5.40 & $n P-i A$ & {$[3]$} \\
\hline 1972 & Smith & $\mathrm{AR}$ & 2.3 & 5.159 & +0.061 & 5.31 & $\mathrm{nP}-\mathrm{iA}$ & {$[3]$} \\
\hline 1972 & Smith & ARC ball & 2.0 & 4.996 & +0.061 & 5.10 & $\mathrm{nP}-\mathrm{iA}$ & {$[3]$} \\
\hline 1972 & Smith & disk & 2.4 & 5.475 & +0.063 & 5.62 & nP-iA & {$[3]$} \\
\hline 1972 & Smith & $\mathrm{CY}$ & 1.9 & 5.469 & +0.059 & 5.59 & $\mathrm{nP}-\mathrm{iA}$ & {$[3]$} \\
\hline 1972 & Smith & disk from $\mathrm{S}$ & 2.3 & 5.036 & +0.063 & 5.19 & $\mathrm{nP}-\mathrm{iA}$ & {$[3]$} \\
\hline 1973 & Brandt and Zarubina & - & 18 & - & c. $a+0.1$ & 7.2 & - & {$[25]$} \\
\hline 1995 & Akahama et al. & SP, high purity & 50 & 5.3 & +0.096 & 9.5 & pyrophyllite & {$[26]$} \\
\hline 2000 & Ishizuka et al. & $\mathrm{SP}$ & 120 & 5.3 & +0.1 & 17.2 & none & {$[27]$} \\
\hline 2019 & present work & $\operatorname{HPT}(N=0)$ & 5.0 & 5.42 & +0.12 & $5.9(5 \mathrm{GPa})$ & Ap-J & - \\
\hline 2019 & present work & $\operatorname{HPT}(N=10)$ & 32.0 & 5.41 & +0.12 & $10.4(32 \mathrm{GPa})$ & Ap-J & - \\
\hline
\end{tabular}

respectively. Previous studies of $\mathrm{HP}$ compression for $\mathrm{V}, \mathrm{Nb}$, and Ta are summarized in Tables 1, 2, and 3, respectively. All of three are considered to belong to a group that exhibits the increase in $T_{\mathrm{c}}$ under the HP circumstances. ${ }^{22)}$ Indeed, smooth linear-increase in $T_{\mathrm{c}}$ is observed only in $\mathrm{V}$. All previous studies for $\mathrm{V}$ have reported the positive $\mathrm{d} T_{\mathrm{c}} / \mathrm{d} P$ coefficient. ${ }^{3,23-27)}$ According to the study of Struzhkin et al., nonstrained $\mathrm{Nb}$ shows the increase in $T_{\mathrm{c}}$ after its slight decrease, 
Table 2 Previous studies on HP experiments for Nb. PTM: pressure-transmitting medium; nP-iA: 1:1 mixture of n-pentane and isoamyl alcohol, Ap-J: Apiezon-J oil, MEW: methanol-ethanol-water mixture in volume ratio of 16:3:1, and $\mathrm{O}_{2}$ : condensed oxygen molecule. CY: cylindrical sample, AR: as-received sample, S: single-crystal, PO: polycrystal, SP: small pieces, PU: purified near the melting point, ARC: arc-melted, and HPT: high-pressure torsion. $N$ denotes the revolution number of the HPT procedure performed at $6 \mathrm{GPa}$.

\begin{tabular}{|c|c|c|c|c|c|c|c|c|c|}
\hline year & authors & sample & $\begin{array}{l}\max . P \\
{[\mathrm{GPa}]}\end{array}$ & $\begin{array}{c}T_{\mathrm{c}}(P=0) \\
{[\mathrm{K}]}\end{array}$ & $\begin{array}{c}\text { initial } \mathrm{d} T_{\mathrm{c}} / \mathrm{d} P \\
{[\mathrm{~K} / \mathrm{GPa}]}\end{array}$ & $\begin{array}{c}\Delta T_{\mathrm{C}} \\
{[\mathrm{K}]}\end{array}$ & $\begin{array}{c}\max . T_{\mathrm{C}} \\
{[\mathrm{K}]}\end{array}$ & PTM & ref \\
\hline 1966 & Gardner and Smith & $\mathrm{CY}$ of $\mathrm{AR}$ & ca. 1 & $9.43,9.52$ & ca. 0 & - & - & - & {$[23]$} \\
\hline 1966 & Gey and Heyden & - & 2.5 & $9.4,9.5$ & ca. -0.03 & - & - & - & {$[29]$} \\
\hline 1968 & Köhnlein & - & 4.1 & 9.40 & +0.012 & 0.31 & $9.71(4 \mathrm{GPa})$ & - & {$[24]$} \\
\hline 1969 & Gey & $\mathrm{PU}$ & 7.3 & - & +0.11 & 0.23 & - & - & {$[30]$} \\
\hline 1970 & Brandt and Papp & $\mathrm{CY}$ & 1.6 & 9.1 & - & $<0.1$ & - & kerosene-oil & {$[31]$} \\
\hline 1970 & Smith & $\mathrm{S}, \mathrm{PO}$ & 2.4 & $9.15-9.21$ & $-0.016--0.025$ & -0.05 & - & $n P-i A$ & {$[32]$} \\
\hline 1997 & Struzhkin et al. & $\mathrm{SP}$ & 70 & ca. 9.2 & -0.025 & 0.7 & $9.9(10 \mathrm{GPa})$ & $\mathrm{NaCl}$ & {$[28]$} \\
\hline 1997 & Struzhkin et al. & $\mathrm{SP}$ & 132 & ca. 9.2 & - & 0.7 & $9.8(15 \mathrm{GPa})$ & none & {$[28]$} \\
\hline 2019 & Mito et al. & $\mathrm{AR}$ & 15.0 & 9.35 & +0.09 & 0.38 & $9.78(15.0 \mathrm{GPa})$ & Ap-J & {$[33]$} \\
\hline 2019 & Mito et al. & $\mathrm{ARC}$ & 6 & 9.05 & +0.14 & 0.26 & 9.31 (ca. $2 \mathrm{GPa})$ & Ap-J, MEW & {$[33]$} \\
\hline 2019 & Mito et al. & $\operatorname{HPT}(N=1)$ & 6.0 & 9.35 & +0.61 & 0.49 & $9.84($ ca. $1 \mathrm{GPa})$ & Ap-J & {$[33]$} \\
\hline 2019 & Mito et al. & $\operatorname{HPT}(N=2)$ & 14.6 & ca. 9.35 & +0.33 & 0.38 & $9.79(14.6 \mathrm{GPa})$ & Ap-J & {$[33]$} \\
\hline 2019 & Mito et al. & $\operatorname{HPT}(N=5)$ & 10.1 & ca. 9.35 & +0.18 & 0.36 & $9.72($ ca. $2 \mathrm{GPa})$ & Ap-J & {$[33]$} \\
\hline 2019 & Mito et al. & $\operatorname{HPT}(N=10)$ & 14.6 & ca. 9.35 & +0.29 & 0.57 & $9.92(2.2 \mathrm{GPa})$ & Ap-J & {$[33]$} \\
\hline 2019 & Mito et al. & $\operatorname{HPT}(N=10)$ & 6.2 & ca. 9.35 & +0.19 & 0.43 & $\geq 9.78(6.2 \mathrm{GPa})$ & MEW & {$[33]$} \\
\hline 2019 & Mito et al. & $\operatorname{HPT}(N=10)$ & 4.3 & ca. 9.35 & +0.03 & 0.08 & $9.43(3.5 \mathrm{GPa})$ & $\mathrm{O}_{2}$ & {$[33]$} \\
\hline 2019 & Mito et al. & $\operatorname{HPT}(N=10)($ cryo $)$ & 5.0 & 9.54 & +0.18 & 0.38 & $9.92(2.8 \mathrm{GPa})$ & Ap-J & {$[33]$} \\
\hline 2019 & Mito et al. & $\mathrm{HPT}(N=20)$ & 5.6 & ca. 9.35 & +0.24 & 0.50 & $9.85(2.4 \mathrm{GPa})$ & Ap-J & {$[33]$} \\
\hline
\end{tabular}

Table 3 Previous studies on HP experiments for Ta. PTM: pressure-transmitting medium; Ap-J: Apiezon-J oil, SP: small pieces, and HPT: high-pressure torsion. $N$ denotes the revolution number of the HPT procedure performed at $6 \mathrm{GPa}$.

\begin{tabular}{|c|c|c|c|c|c|c|c|c|}
\hline year & authors & sample & $\begin{array}{c}\max . P \\
{[\mathrm{GPa}]}\end{array}$ & $\begin{array}{c}T_{\mathrm{c}}(P=0) \\
{[\mathrm{K}]}\end{array}$ & $\begin{array}{l}\text { initial } \mathrm{d} T_{\mathrm{c}} / \mathrm{d} P \\
{[\mathrm{~K} / \mathrm{GPa}]}\end{array}$ & $\begin{array}{c}\max . T_{\mathrm{c}} \\
{[\mathrm{K}]}\end{array}$ & PTM & ref \\
\hline 1956 & Hatton & moderately annealed & 0.5 & 4.385 & $-0.025 \sim-0.053$ & - & - & {$[34]$} \\
\hline 1958 & Jennings and Swenson & $2300 \mathrm{C}^{\circ}$ annealed & ca. 1 & 4.305 & -0.026 & - & solid $\mathrm{H}_{2}$ & {$[2]$} \\
\hline 1968 & Kohnlein & - & 3.6 & 4.43 & -0.026 & - & - & {$[24]$} \\
\hline 1997 & Struzhkin et al. & SP & 43 & 4.43 & -0.022 & $4.5(43 \mathrm{GPa})$ & none & {$[28]$} \\
\hline 2019 & present work & $\operatorname{HPT}(N=0)$ & 6.0 & 4.40 & +0.045 & 4.44 & Ap-J & - \\
\hline 2019 & present work & $\mathrm{HPT}(N=0.5)$ & 9.5 & 4.367 & +0.004 & 4.372 & Ap-J & - \\
\hline 2019 & present work & $\operatorname{HPT}(N=2)$ & 4.7 & 4.42 & +0.015 & 4.45 & Ap-J & - \\
\hline 2019 & present work & $\operatorname{HPT}(N=5)$ & 6.9 & 4.37 & -0.058 & 4.41 & Ap-J & - \\
\hline 2019 & present work & $\mathrm{HPT}(N=10)$ & 8.8 & 4.36 & +0.056 & 4.42 & Ap-J & - \\
\hline
\end{tabular}

and has the optimal $T_{\mathrm{c}}$ of $9.9 \mathrm{~K}$ at around $P=10 \mathrm{GPa}{ }^{28)}$ Indeed, the initial $\mathrm{d} T_{\mathrm{c}} / \mathrm{d} P$ for $\mathrm{Nb}$ depends on experimental conditions such as the sample condition and kind of pressure transmitting medium (PTM). ${ }^{23,24,28-33)}$ Contrary to the above two, Ta exhibits the decrease in $T_{\mathrm{c}}$ over wide pressure range, $2,24,28,34,35)$ and there is only one plot suggesting the increase in $T_{\mathrm{c}}$ at $43 \mathrm{GPa}^{28)}$ Thus, there are much variety in the pressure dependence of $T_{\mathrm{c}}$ among $\mathrm{V}, \mathrm{Nb}$, and Ta. We are exploring the observation of new pressure response using the SPD materials. In the present study, we conduct the HP compression to strained materials subjected to the HPT process (see Fig. 2), considered as the so-called Josephson arrays of small grains, and control both Josephson coupling between superconducting grains and lattice strain at the unitcell level. Figure 3 shows a digest of the present review. There are two parameters such as $N$ related with the initially installed strain [Fig. 2(a)] and pressure $(P)$ related with additional strain [Fig. 2(b)]. Both HPT apparatus and HP one commonly using anvils have the same route of Bridgman's excellent achievement. ${ }^{13)}$ 

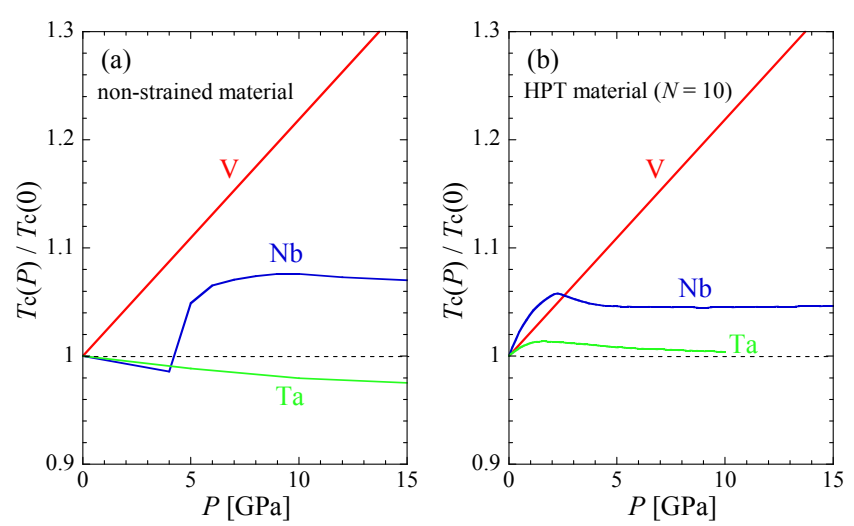

Fig. 3 Digest of the present studies for fifth-element transition-metal superconductors, $\mathrm{V}, \mathrm{Nb}$, and $\mathrm{Ta}$. The pressure dependences of $T_{\mathrm{c}}$ normalized with the value at ambient pressure, $T_{\mathrm{c}}(0)$, for $P \leq 15 \mathrm{GPa}$ are presented in two cases of (a) non-strained material and (b) material subjected to the HPT procedure with $N=10$ at $6 \mathrm{GPa}$.

\section{Experimentals}

\subsection{High-pressure torsion (HPT)}

As illustrated in Fig. 2(a), the disks of $\mathrm{V}, \mathrm{Nb}$, and Ta were subjected to HPT processing at room temperature under a selected pressure of $P=6 \mathrm{GPa}$ and revolutions of $N$ up to 20 with a rotation speed of $1 \mathrm{rpm}$. Specimens with dimensions of approximately $0.8 \times 0.8 \times 0.5 \mathrm{~mm}^{3}$ were cut from an HPT-processed disk using a wirecutting electric discharge machine at the position of $2.5 \mathrm{~mm}$ from the disk center. For instance, in $\mathrm{Nb}$, we remark that for $N<2$, dislocations are stored as illustrated in Fig. 2(a-1), and the grain size largely changes. ${ }^{36)}$ The small-angle grain boundaries formed in these cases gradually transform to large-angle grain boundaries with increasing $N$ (corresponding to the change form Fig. 2(a-2) to Fig. 2(a-3)). On the other hand, for $N \geq 2$, the fraction of large-angle grain boundaries increases due to annihilation of dislocations at grain boundaries (illustrated in Fig. 2(a-3)), leading to that the grain size remains unchanged because of the balance between dislocation generation by straining and the dislocation annihilation at grain boundaries. The grain size at steady state for $\mathrm{V}, \mathrm{Nb}$, and $\mathrm{Ta}$ is $330 \mathrm{~nm}$, $240 \mathrm{~nm}$, and $180 \mathrm{~nm}$, respectively. ${ }^{37}$ )

In $\mathrm{Nb}$, we note here that the average grain sizes for $\mathrm{AR}$, $N=2, N=5$, and $N=10$ (cryo) samples have been reported to be $140 \mu \mathrm{m}, 250 \mathrm{~nm}, 240 \mathrm{~nm}$, and ca. $40 \mathrm{~nm}$, respectively. ${ }^{36,38)}$ Thus, the shear insertion leads to a significant reduction in the grain size. The superconducting coherence length $\xi(0)$ for the single-crystal has been reported as around $20 \mathrm{~nm},{ }^{39)}$ and the aforementioned grain sizes for $N=2$, $N=5$, and $N=10$ (cryo) are at a level comparable with $\xi(0) .{ }^{10)}$ The penetration depth for the single-crystal is approximately $40 \mathrm{~nm},{ }^{39)}$ and large superconducting signal observed in the present study reflects the intergrain coupling via grain boundaries. At ambient pressure, $T_{\mathrm{c}}$ for $\mathrm{AR}$ is approximately $9.25 \mathrm{~K}$, and $T_{\mathrm{c}}$ exhibits a maximum of approximately $9.37 \mathrm{~K}$ at $N=2$. In addition, $T_{\mathrm{c}}$ remains unchanged at $9.35 \mathrm{~K}$ for $N>10 .^{10)}$

For the following hydrostatic experiment, small pieces with side lengths less than $0.1 \mathrm{~mm}$ were prepared from the specimens with dimensions of approximately $0.8 \times 0.8 \times$
$0.5 \mathrm{~mm}^{3}$ as illustrated in Fig. 2. As for Ta, it has been known that Ta subjected to the HPT processing has two superconducting transitions. ${ }^{40)}$ The Meissner signal with lower $T_{\mathrm{c}}$ exhibits majority of superconducting volume fraction. In the present experiment using little sample volume, it was difficult to pursue the change in the Meissner signal with higher $T_{\mathrm{c}}$ under HP compression. Thus, for HPT-Ta, we have paid much attention to the change in the Meissner signal with lower $T_{\mathrm{c}}$ that exhibits the same pressure dependence as that for the midpoint of the Meissner signal. In the electrical resistance measurements for superconductors under high pressure, it is difficult to measure zero resistance. In particular, this problem becomes more serious in strained superconductors under non-HP compression. Therefore, we adopt the Meissner measurements to evaluate $T_{\mathrm{c}}$ of $\mathrm{V}, \mathrm{Nb}$, and $\mathrm{Ta}$ in the present extreme condition.

\subsection{SQUID magnetometer with miniature-DAC (mDAC)}

The AC magnetization of the materials at pressures of GPa level was measured using a superconducting quantum interference device (SQUID) magnetometer equipped with an AC option. ${ }^{41-47)}$ The frequency and amplitude of the AC field were $10 \mathrm{~Hz}$ and $3.9 \mathrm{Oe}$, respectively. At small amplitude of AC field, the magnitude of in-phase one $\left(m^{\prime}\right)$ is almost consistent with the Meissner signal observed at static magnetic field. ${ }^{48)}$ Sufficiently below $T_{\mathrm{c}}$, there is no out-ofphase component reflecting energy loss, and $m^{\prime}$ is related with the volume fraction of magnetic shielding. The onset temperature of the superconducting signal was evaluated as the confident $T_{\mathrm{c}}$ after the quantitative deviation of the onset temperature was confirmed to be consistent with that of the midpoint temperature.

Contraction corresponding to a stress of up to $15 \mathrm{GPa}$ was achieved using a miniature $\mathrm{CuBe}$ diamond anvil cell (DAC) that consisted of two diamond anvils with flat tips having a diameter of $0.5 \mathrm{~mm}$ and a 0.25 -mm-thick Re gasket. ${ }^{49,50)}$ In the HP-type experiments, liquid-like PTM, Apiezon-J oil (Ap-J), was confined together with small pieces of the HPT materials in the sample chamber: At room temperature, Apiezon-J oil solidifies at a few one-tenth GPa. The pressure value at room temperature $\left(P_{\mathrm{rt}}\right)$ was evaluated by measuring the fluorescence of ruby ${ }^{51)}$ located in the sample cavity with the HPT materials. According to Ref. 49, when temperature is cooled from room temperature to liquid helium temperature, there appears the pressure increase equal to approximately $10 \%$ of $P_{\mathrm{rt}}$. In this paper, $P_{\mathrm{rt}}$ is adopted as the pressure value $(P)$ in each measurement, since the comparison between magnetic and structural data at low pressures of $P<7 \mathrm{GPa}$ is meaningful.

\subsection{SQUID-based vibrating coil magnetometer (SQUID- VCM)}

The DAC is mounted in the ${ }^{4} \mathrm{He}$-refrigerator with a $1 \mathrm{~K}$ pot, and the entire DAC is heated. The use of diamond anvils with different culet size (i.e. 500 and $550 \mu \mathrm{m}$ ) allows the $\mathrm{CuBe}-\mathrm{NiCrAl}$ composite gasket to be deformed away from the NbTi detection coil. The NiCrAl disk with the diameter of $410 \mu \mathrm{m}$ was inserted into the hole drilled in the center of CuBe gasket. $^{52)}$ After pressing the composite gasket down to 
$70 \mu \mathrm{m}$, a hole with the diameter of $200 \mu \mathrm{m}$ was drilled in the center. In the sample cavity, the small pieces of HPT-V $(N=10)$ were held together with Apiezon-J oil and ruby as a room-temperature manometer. The magnitude of the pressure $(P)$ was estimated at room temperature by measuring the fluorescence of ruby. ${ }^{51)}$

Using an actuating unit consisting of three actuators, the detection coil is ideally vibrated at the place where the slope of magnetic flux against the distance from the sample is maximum. ${ }^{53)}$ To obtain sufficient amplitude of the vibration of the detection coil at liquid helium temperature, the driving frequency of the vibrating actuators, consisting of bimorph type piezoelectric actuators, was chosen to be $168 \mathrm{~Hz}$ near the resonating frequency of the vibrating actuators. ${ }^{54}$ The DAC and the actuating unit are located in the cryostat as shown elsewhere. ${ }^{54)} H_{\mathrm{dc}}$ was applied at $30 \mathrm{Oe}$ at maximum. The use of a lock-in amplifier at the vibration frequency yields the amplitude of $V_{\mathrm{VCM}}$.

\subsection{X-ray diffraction under hydrostatic compression}

In order to determine the structural shrinkage under pressure, we performed X-ray diffraction (XRD) analyses for $\mathrm{Nb}$ and $\mathrm{Ta}$ under high pressure of up to $P=6.8 \mathrm{GPa}$ at room temperature using a synchrotron radiation XRD system with a cylindrical imaging plate at the Photon Factory at the Institute of Materials Structure Science, High Energy Accelerator Research Organization. ${ }^{55)}$ The energy of the incident X-rays was $16 \mathrm{keV}$. Pressure was applied using a DAC that consisted of two diamond anvils with flat tips having a diameter of $0.8 \mathrm{~mm}$ and a $0.3-\mathrm{mm}$-thick $\mathrm{CuBe}$ gasket. The small pieces of HPT-Nb or HPT-Ta were placed in a randomly oriented manner in a sample cavity with a diameter of $0.4 \mathrm{~mm}$ along with a ruby of manometer and transparent PTM such as fluorinated oil (FC77, Sumitomo 3M Ltd.). Transparent FC77 undergoes solidification below $1 \mathrm{GPa}$ at room temperature ${ }^{56)}$ similarly to brown Ap-J. All the atomic positions in the body-centered cubic (bcc) structure are special positions, and therefore, the structural parameters can be evaluated just with the use of the diffraction peak angle. The lattice parameters were estimated using all of observed diffraction peaks: For $\mathrm{Nb}$, we used five diffraction peaks of the plane indices (110), (200), (211), (220), and (310). For Ta, the diffraction peak of the plane index (310) was not used.

\section{Experimental Results}

\subsection{HPT-V}

Figure 4 shows the temperature dependence of in-phase ac magnetization $m^{\prime}$ for HPT-V $(N=0)$ (a) and HPT-V $(N=10)$ (b) by using a SQUID magnetometer with miniature-DAC (mDAC). Figure 5 shows $V_{\mathrm{VCM}}$ that corresponds to the temperature dependence of dc magnetic susceptibility for HPT-V $(N=10)$ by using the SQUID-VCM magnetometer. Figure 6 shows the pressure dependence of $T_{\mathrm{c}}$ for HPT-V $(N=0)$ and HPT-V $(N=10)$. Comparing $T_{\mathrm{c}}$ 's at $P=5.0 \mathrm{GPa}$, HPT-V $(N=10)$ has slightly higher value than HPT-V $(N=0)$. However, when we see their data in wider pressure range, there is no prominent difference in the change in $T_{\mathrm{c}}$ between HPT-V $(N=0)$ and HPT-V $(N=10)$, which are consistent with the previous data by Ishizuka et al. ${ }^{27)}$
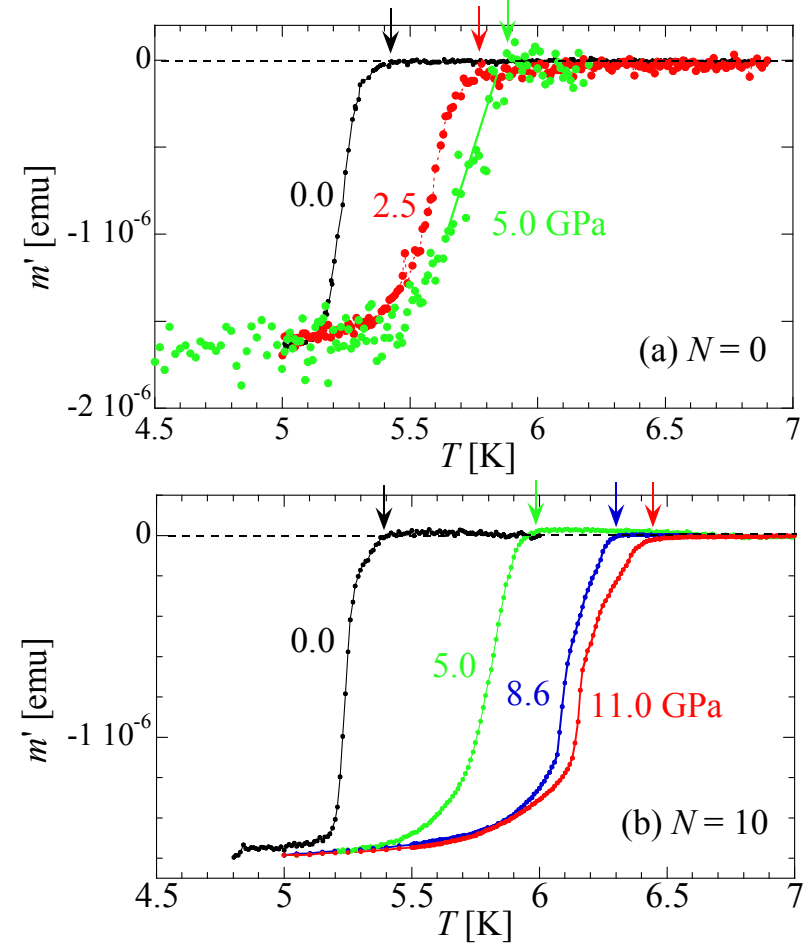

Fig. 4 Temperature dependence of in-phase ac magnetization $m^{\prime}$ for HPT$\mathrm{V}(N=0)(\mathrm{a})$ and HPT-V $(N=10)(\mathrm{b})$.

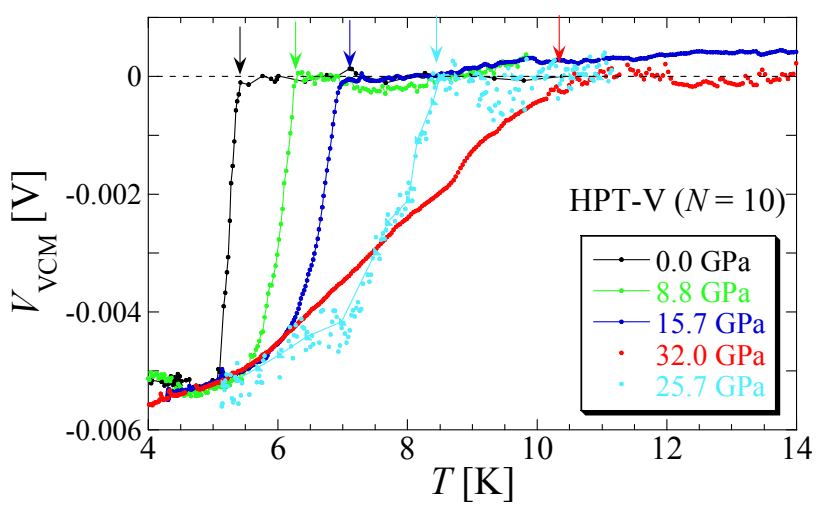

Fig. 5 Temperature dependence of dc magnetic susceptibility for HPT-V $(N=10)$.

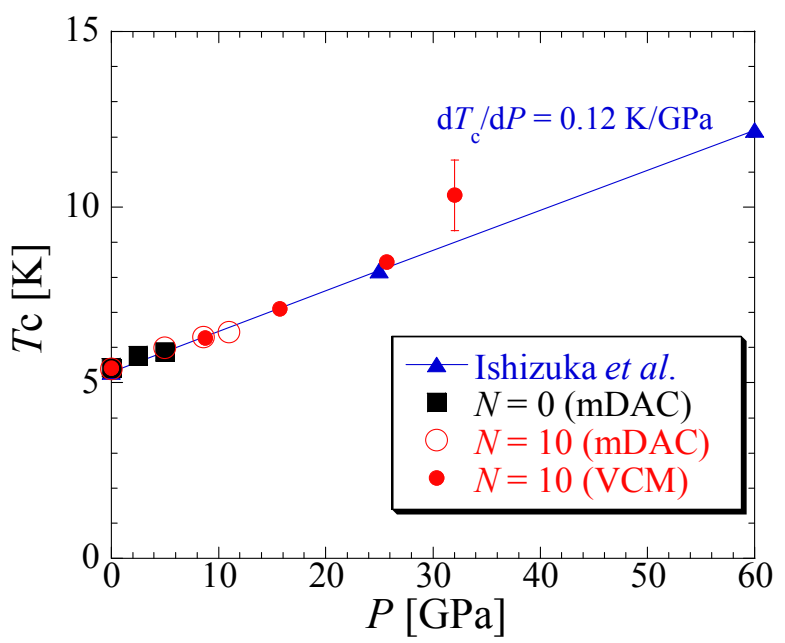

Fig. 6 Pressure dependence of superconducting transition temperature $T_{\mathrm{c}}$ for HPT-V $(N=0)$ and HPT-V $(N=10)$. For reference, the previous data for non-strained $\mathrm{V}$ are also presented. ${ }^{27}$ ) 

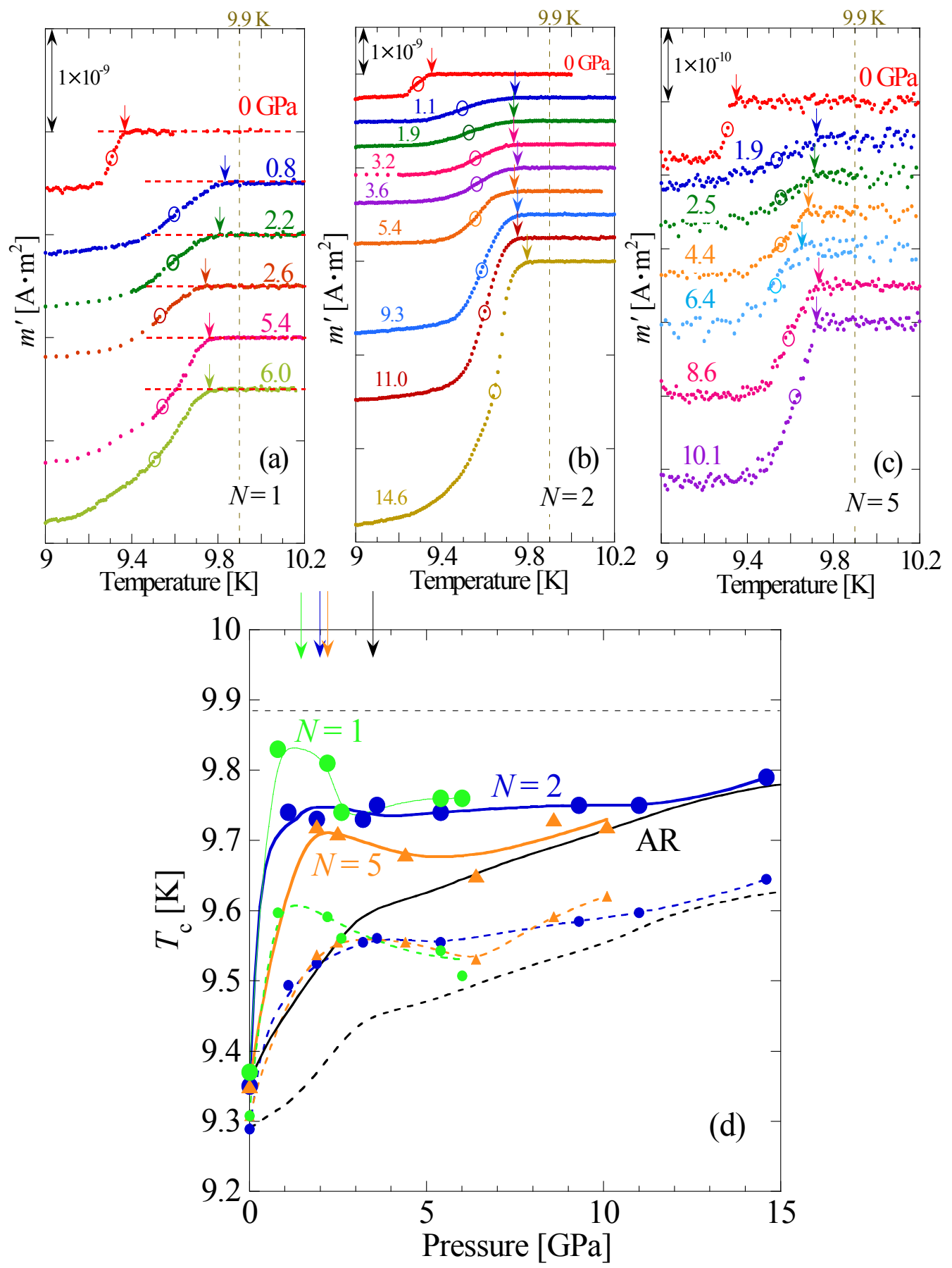

Fig. 7 Temperature dependence of in-phase ac magnetization $m^{\prime}$ for HPT-Nb samples with $N=1$ (a), 2 (b) and 5 (c) at various pressures. $^{33)}$ The diameter of the sample chamber for $N=1$ and 2 was $0.2 \mathrm{~mm}$, and that for $N=5$ was $0.1 \mathrm{~mm}$. The onset of the superconducting signal is presented with an arrow, and the midpoint is marked with an open circle. (d) Pressure dependence of superconducting transition temperature $T_{\mathrm{c}}$ for HPT-Nb for $N=1,2$, and 5. Arrows represent the threshold pressure $\left(P_{\mathrm{c}}\right)$. For three samples, Ap-J was used as the PTM. For reference, the guide curves corresponding to $T_{\mathrm{c}}(P)$ of the AR sample with Ap-J as the PTM (see Ref. 33) are also presented with the black solid (onset) and broken (midpoint) curves.

\subsection{HPT-Nb}

Figures 7(a)-(c) shows the temperature dependence of inphase ac magnetization $m^{\prime}$ for the HPT-Nb $(N=1,2$, and 10) materials by using a SQUID magnetometer with mDAC. The onset of the Meissner signal shows the change similar to that of the midpoint, and the onset temperature is determined to be $T_{\mathrm{c}}$. The pressure dependence of $T_{\mathrm{c}}$ for HPT-Nb $(N=1,2$, and 10) is shown in Fig. 7(d), comparing to that of asreceived (AR) $\mathrm{Nb}$ material. Three HPT materials exhibit the larger enhancement of $T_{\mathrm{c}}$ than the AR material. In particular, HPT-Nb $(N=1)$ shows the increase in $T_{\mathrm{c}}$ over $9.8 \mathrm{~K}$ at around $P=1 \mathrm{GPa}$. As $N$ increases like $N=1 \rightarrow 5$, the maximum $T_{\mathrm{c}}$ shifts toward higher pressure side, decreasing the maximum value.

Figures 8(a)-(c) shows the temperature dependence of inphase ac magnetization $m^{\prime}$ for the HPT-Nb $(N=10)$ material by using a SQUID magnetometer with $\mathrm{mDAC}$. The pressure dependence of the onset temperature $T_{\mathrm{c}}$ for HPT-Nb $(N=10)$ 

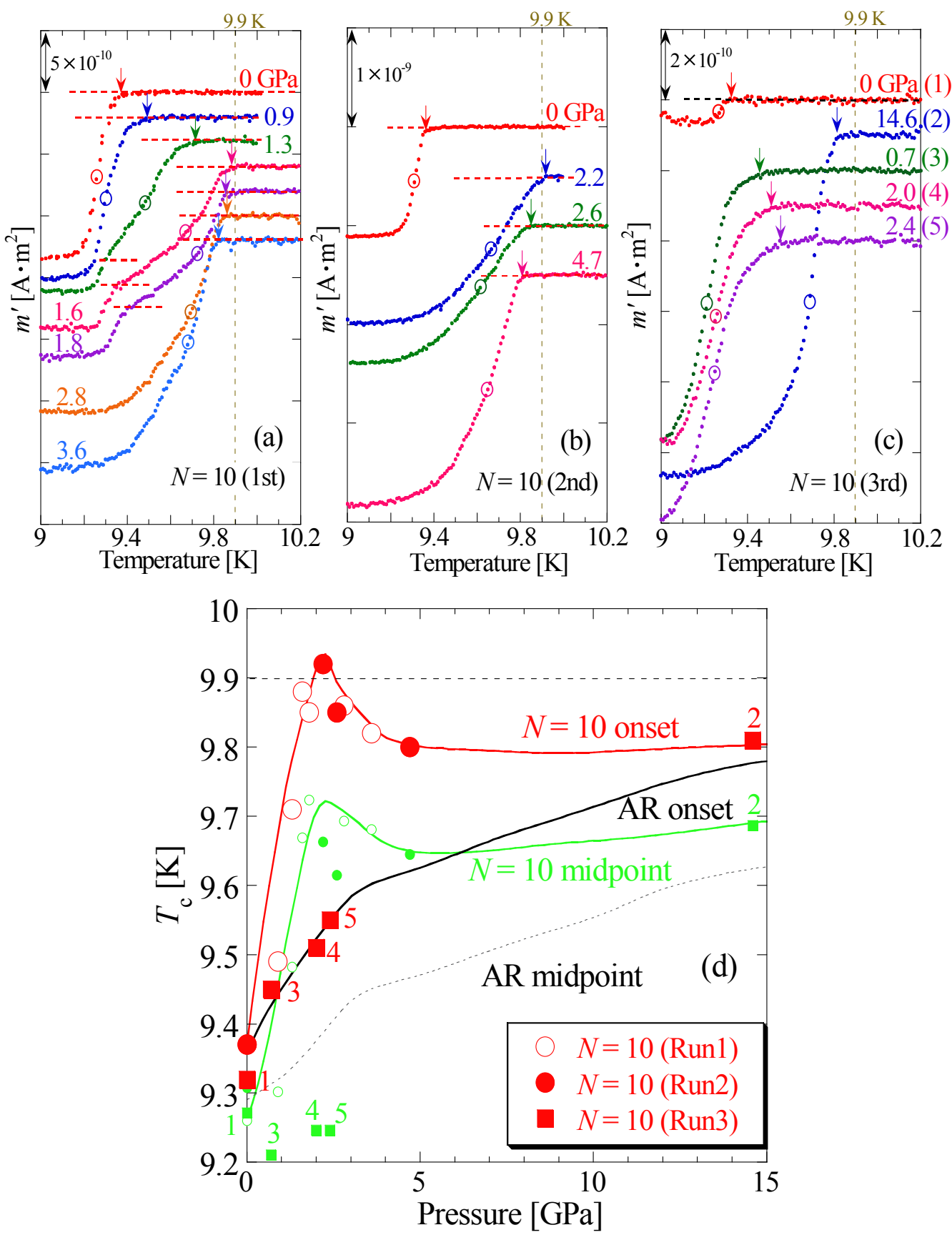

Fig. 8 Temperature dependence of in-phase ac magnetization $m^{\prime}$ for HPT-Nb samples with $N=10$ using Ap-J as PTM [first run (a), second one (b), and third one (c)] at various pressures. ${ }^{33)}$ The diameter of the sample chamber for the first and second runs was $0.2 \mathrm{~mm}$, and that for the third run was $0.1 \mathrm{~mm}$. The onset of the superconducting signal is presented with an arrow, and the midpoint is marked with an open circle. For $P=1.3,1.6$, and $1.8 \mathrm{GPa}$ in (a), the midpoint was evaluated for the major superconducting signal. The number beside the pressure value in the figure indicates the measurement trial number. (d) Pressure dependence of superconducting transition temperature $T_{\mathrm{c}}$ for HPT-Nb with $N=10$. Here, Ap-J was used as the PTM. The number beside each symbol indicates the measurement trial number. For reference, the guide curves corresponding to $T_{\mathrm{c}}(P)$ of the sample AR using Ap-J as PTM (see Ref. 33) are also presented with the black solid (onset) and broken (midpoint) curves.

is shown in Fig. 8(d). $T_{\mathrm{c}}$ of HPT-Nb $(N=10)$ exhibits the maximum $T_{\mathrm{c}}$ over $9.8 \mathrm{~K}$ at around $P=2 \mathrm{GPa}$. The behavior of $T_{\mathrm{c}}$ for HPT-Nb $(N=10)$ is not on an expanded line of changes for $N=1 \rightarrow 5$. $T_{\mathrm{c}}$ for all HPT-Nb $(N=1,2,5,10)$ materials shows consistency with that for AR at around $P=15 \mathrm{GPa}$. The feature due to the HPT processing appears in the pressure region below $15 \mathrm{GPa}$.

Figures 9 shows the pressure dependence of interplanar distance of (110), (200), (211), (220), and (310) for the HPT$\mathrm{Nb}$ materials for $N=1$ (c) and 10 (d). For reference, the data for both arc-melting (ARC) and AR materials are also shown in Figs. 9(a) and 9(b), respectively. At first, the AR material exhibits anisotropic transformation of the unit cell structure at around $P=2 \mathrm{GPa}$, suggesting that strain accumulated in the cubic lattice with high symmetry is released at a threshold pressure $\left(P_{\mathrm{c}}\right)$. This phenomenon does not occur in the ARC material without residual strain, suggesting the importance of residual strain. When the HPT procedure with $N=1$ is subjected to the AR material, the grain refinement enhances the capacity of accumulating the additional strain, resulting in 

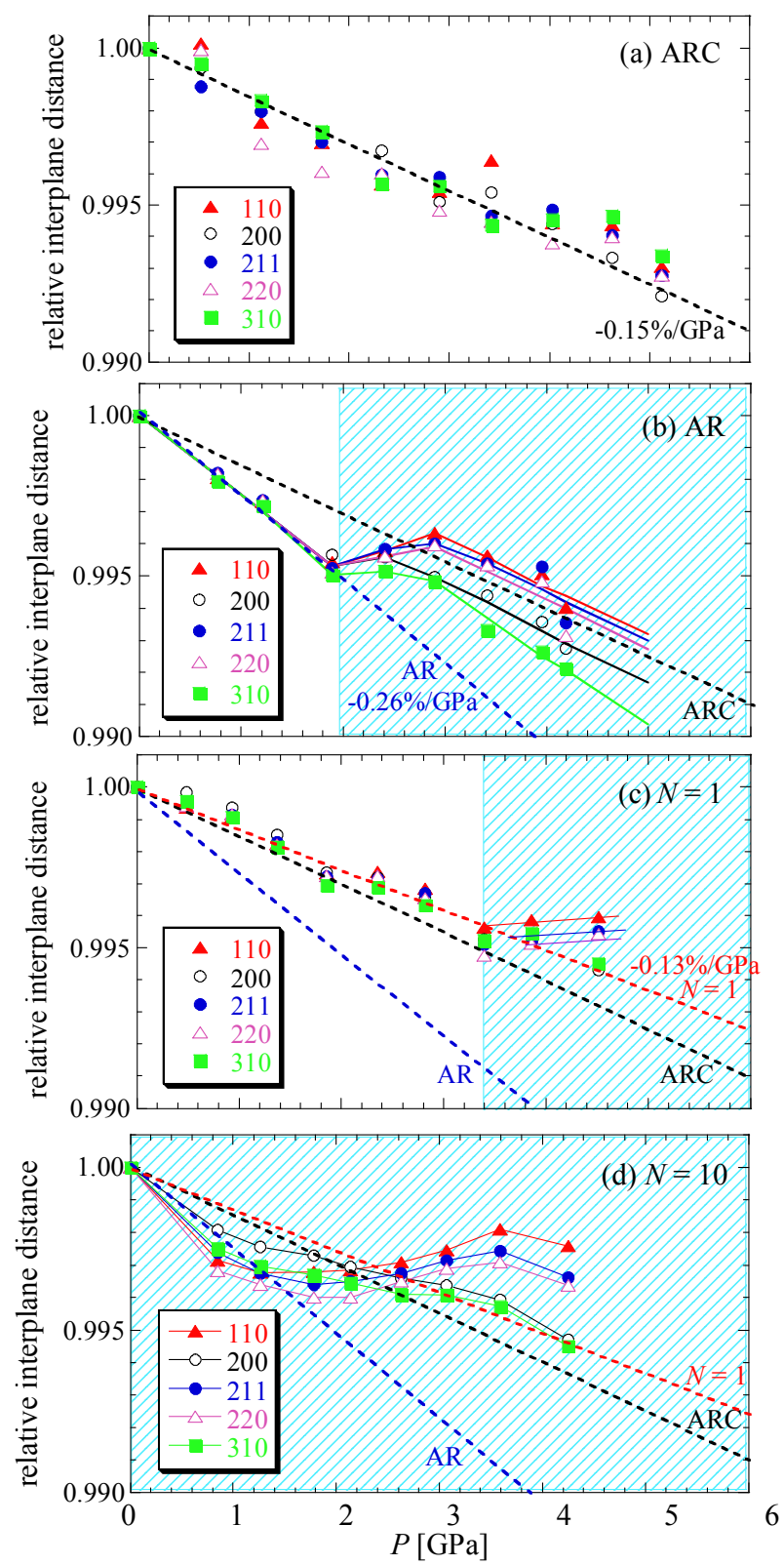

Fig. 9 Pressure dependence of normalized interplanar distance of (110), (200), (211), (220), and (310) planes for ARC (a), AR (b), HPT-Nb $(N=1)(\mathrm{c})$, and HPT-Nb $(N=10)(\mathrm{d}) .{ }^{33)}$ The broken guide lines in all figures represent the interplanar distance change for ARC (black), AR (blue), and $N=1$ (red). These initial slope values in the pressure regime where isotropic structure change occurs are also indicated. The pressure regime corresponding to anisotropic structure change is indicated in lightblue hatching. FC77 was used as PTM in all cases.

the increase in $P_{\mathrm{c}}$ to approximately $3.4 \mathrm{GPa}$. However, HPT with the level of $N=10$ does not permit to accumulate any additional strain, so that anisotropic unit-cell deformation occurs even at quite small pressure.

Figure 10 shows the pressure dependence of anisotropic lattice deformation using normalized lattice constants $a_{1} / a_{\text {cubic }}, a_{2} / a_{\text {cubic }}$, and $a_{3} / a_{\text {cubic }}$ for ARC (a), AR (b), HPT$\mathrm{Nb}(N=1)(\mathrm{c})$, and HPT-Nb $(N=10)(\mathrm{d})$ samples. Herein, $a_{\text {cubic }}$ is a lattice constant considering the bcc lattice. Comparing with ARC, AR and HPT $(N=1)$ exhibits the one-direction shrinkage and two-direction expansion. On the other hand, the HPT $(N=10)$ material exhibits two-direction
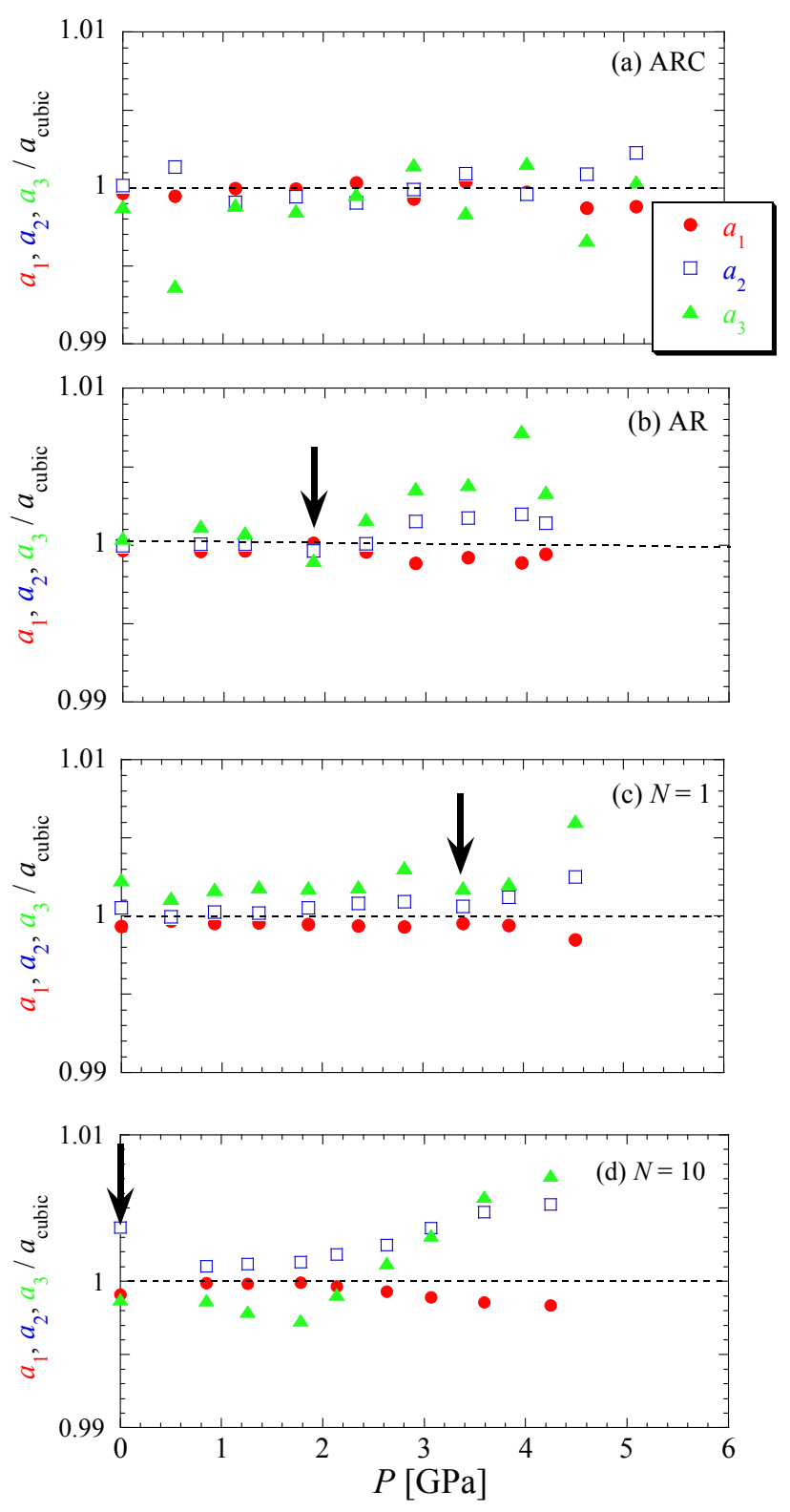

Fig. 10 Pressure dependence of anisotropic lattice deformation using normalized lattice constants $a_{1} / a_{\text {cubic }}, a_{2} / a_{\text {cubic }}$, and $a_{3} / a_{\text {cubic }}$ for ARC (a), AR (b), HPT-Nb $(N=1)(\mathrm{c})$, and HPT-Nb $(N=10)(\mathrm{d}){ }^{33)} a_{\text {cubic }}$ is a lattice constant considering the bcc lattice. The threshold pressure for anisotropic structure change, determined in Fig. 9, is indicated by an arrow. FC77 was used as the PTM in all cases.

shrinkage for $P<2 \mathrm{GPa}$ and two-direction expansion for $P>2 \mathrm{GPa}$. At around $2 \mathrm{GPa}$, the deformation manner changes, suggesting complex manner of strain release. In particular, the HPT $(N=10)$ material with heavy residual strain has a deformed bcc structure, which is close to the body-centered tetragonal (bct) structure for $P>3 \mathrm{GPa}$.

\subsection{HPT-Ta}

Figures 11, 12, and 13 shows the temperature dependence of in-phase ac magnetization $m^{\prime}$ for the HPT-Ta $(N=0,2$, and 10) materials by using a SQUID magnetometer with mDAC. The onset of the Meissner signal is determined to be $T_{\mathrm{c}}$. The pressure dependence of $T_{\mathrm{c}}$ for HPT-Ta $(N=0,2$, and 10 ) is shown in Fig. 14, comparing to the previous data for 


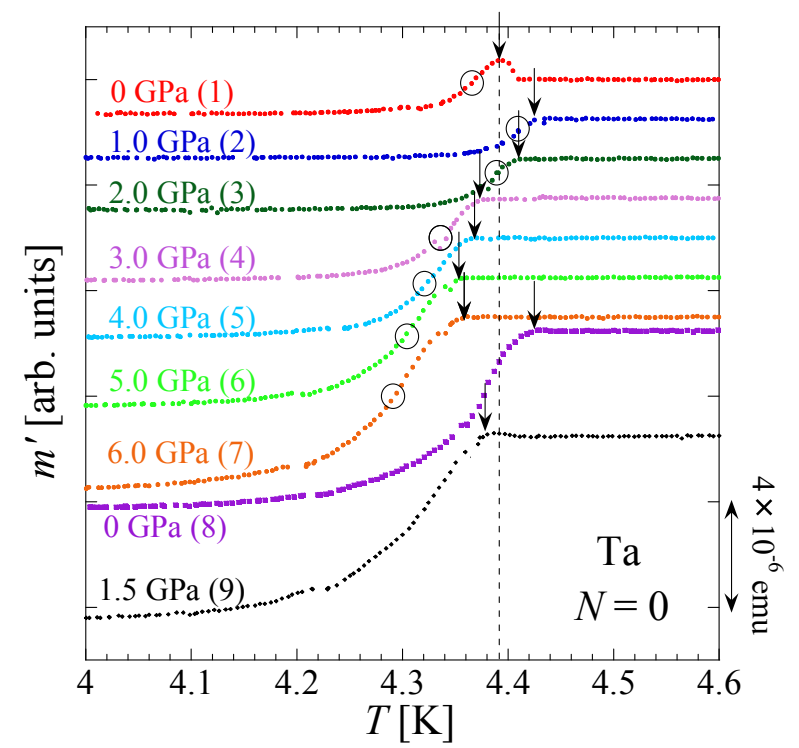

Fig. 11 Temperature dependence of in-phase ac magnetization $m^{\prime}$ for HPTTa with $N=0$ using Ap-J as PTM at various pressures. The diameter of the sample chamber was $0.2 \mathrm{~mm}$. The onset of the superconducting signal is presented with an arrow, and the midpoint is marked with an open circle. The number beside the pressure value in the figure indicates the measurement trial number.

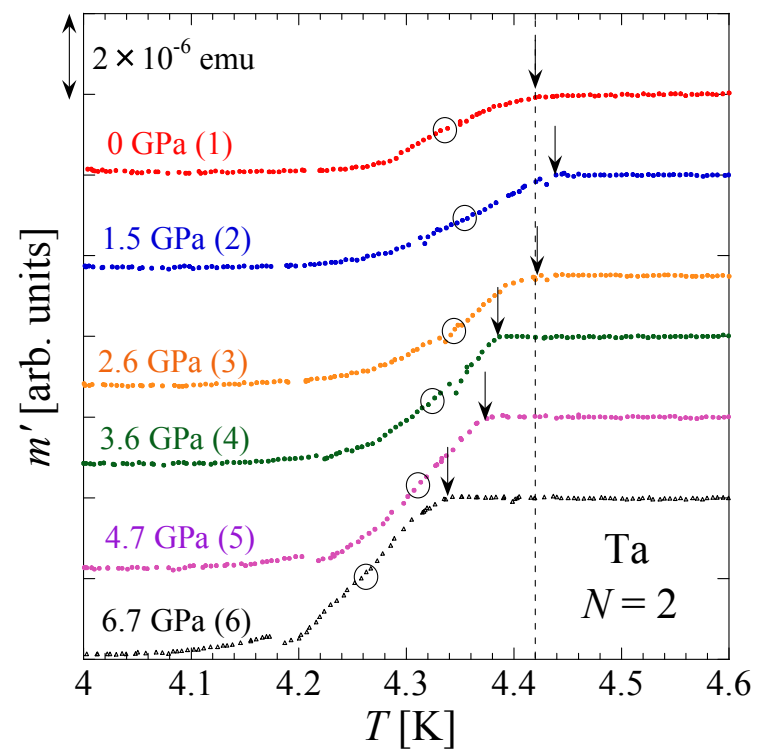

Fig. 12 Temperature dependence of in-phase ac magnetization $m^{\prime}$ for HPTTa with $N=2$ using Ap-J as PTM at various pressures. The diameter of the sample chamber was $0.2 \mathrm{~mm}$. The onset of the superconducting signal is presented with an arrow, and the midpoint is marked with an open circle. The number beside the pressure value in the figure indicates the measurement trial number.

non-strained $\mathrm{Ta}^{28)}$ All of three HPT materials exhibit the maximum $T_{\mathrm{c}}$ at around $P=1-2 \mathrm{GPa}$. For $P<2 \mathrm{GPa}$, HPT $(N=2)$ shows the highest $T_{\mathrm{c}}$ among three, whereas for $P>2 \mathrm{GPa}$, HPT $(N=10)$ does the highest $T_{\mathrm{c}}$. HPT $(N=$ $0.5)$ with initial accumulation of dislocation exhibits little increase in $T_{\mathrm{c}}$. HPT $(N=5)$ with competition of small-angle grain boundaries and large-angle grain boundaries exhibits strange down-up-down-up-down change in $T_{\mathrm{c}}$. These behaviors suggests that intergrain network becomes a key factor to determine $T_{\mathrm{c}}$.

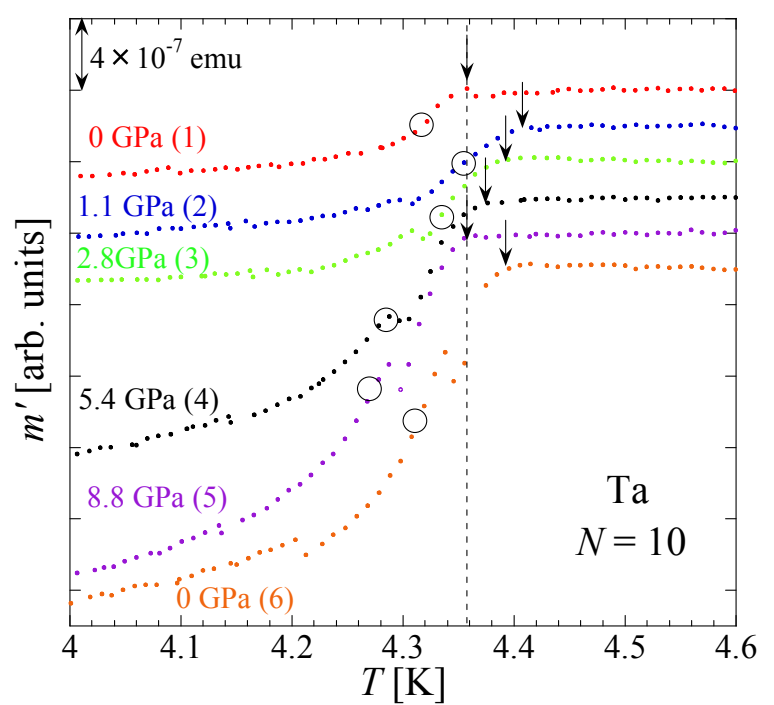

Fig. 13 Temperature dependence of in-phase ac magnetization $m^{\prime}$ for HPTTa with $N=10$ using Ap-J as PTM at various pressures. The diameter of the sample chamber was $0.2 \mathrm{~mm}$. The onset of the superconducting signal is presented with an arrow, and the midpoint is marked with an open circle. The number beside the pressure value in the figure indicates the measurement trial number.

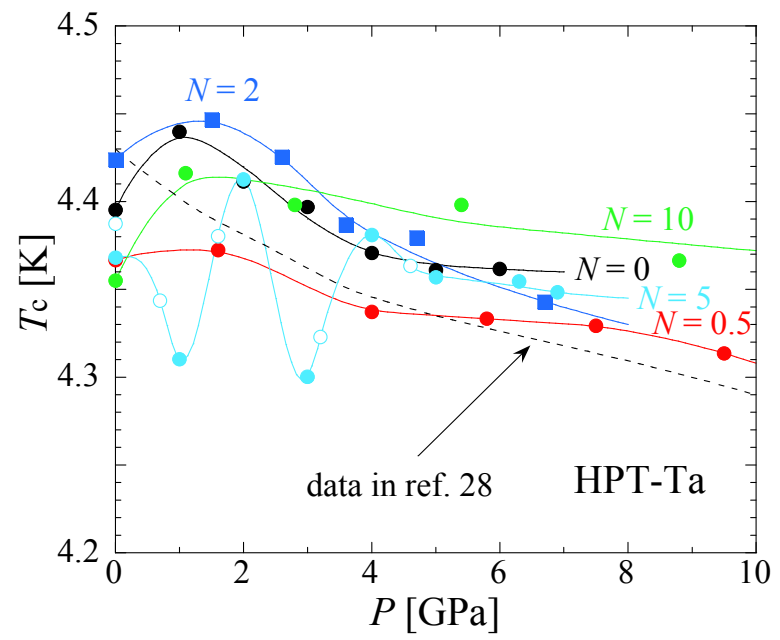

Fig. 14 Pressure dependence of superconducting transition temperature $T_{\mathrm{c}}$ for HPT-Ta $(N=0,0.5,2,5$, and 10) materials. For reference, the previous data for non-strained Ta are also presented with black broken curve. ${ }^{28)}$ For $N=5$, the closed circles stand for the data in the pressureincreasing process up to $6.9 \mathrm{GPa}$, and the open circles do for those in the pressure-increasing process after releasing the pressure of $6.9 \mathrm{GPa}$.

Figures 15 shows the pressure dependence of interplanar distance of (110), (200), (211), and (220) for the HPT-Ta samples for $N=0$ (a), 2 (b), and 10 (c). In all of three, all of interplanar distances exhibit the shrinkage, and there are no anisotropic deformation of the bcc unit cell. For $N=0$, at around $P=1-2 \mathrm{GPa}$, the shrinkage magnitude is suppressed similarly to that observed in AR-Nb. The above pressure region is consistent with that possessing the maximum of $T_{\mathrm{c}}$. On the other hand, both $N=2$ and 10 shows that the uniform contraction continues monotonously. Both HPT $(N=2)$ and HPT $(N=10)$ do not show anomalous change in $a$ at around $P=1-2 \mathrm{GPa}$, suggesting that the increase in $T_{\mathrm{c}}$ cannot be explained only with the change in lattice constant. Figure 16 shows the pressure dependence of normalized lattice constant 

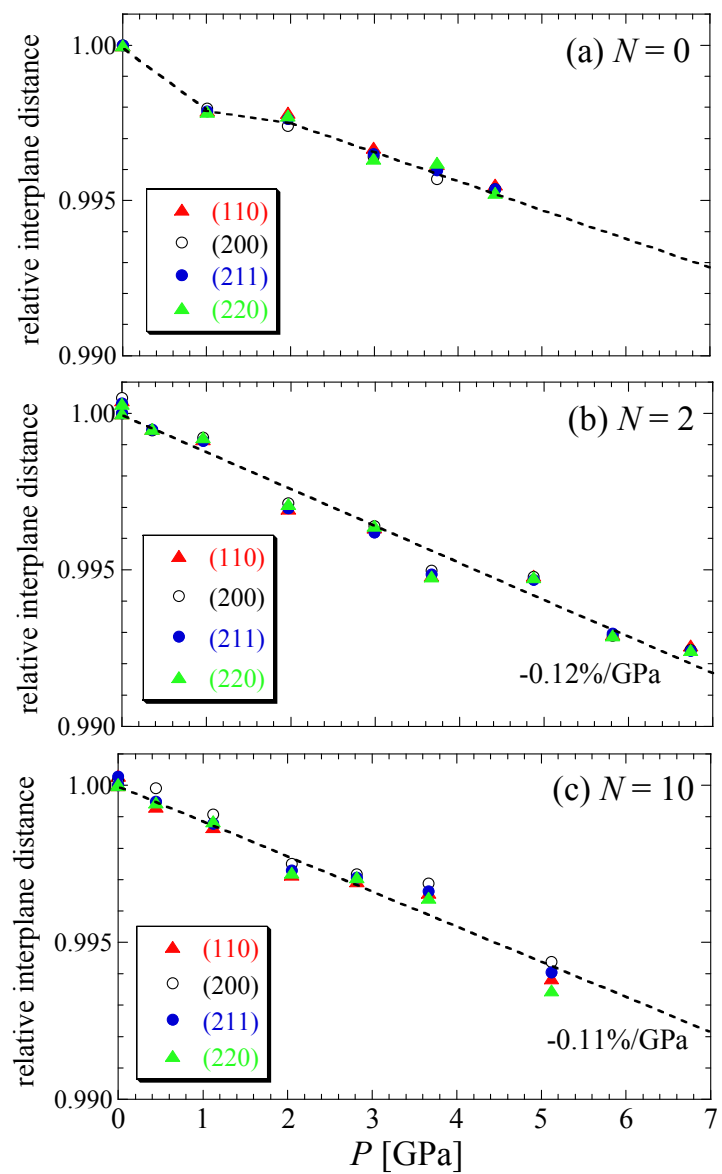

Fig. 15 Pressure dependence of normalized interplanar distance of (110), (200), (211), and (220) planes for HPT-Ta with $N=0$ (a), 2 (b), and 10 (c).

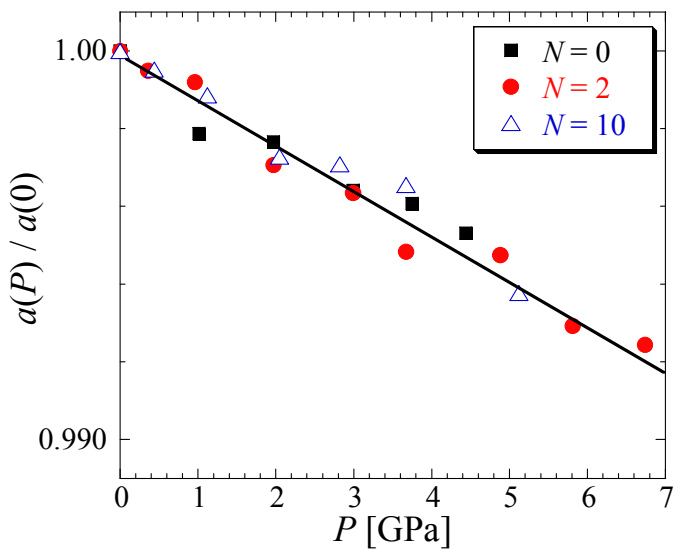

Fig. 16 Pressure dependence of lattice constant of the bcc lattice for HPT-Ta with $N=0,2$, and 10 . The lattice constant $a$ is normalized with the value at ambient pressure. The solid line has the slope of $-1.2 \times$ $10^{-3} \mathrm{~K} / \mathrm{GPa}$

$a$. There is no prominent $N$ dependence, and $a$ continues to decrease monotonously in the considered pressure region.

\section{Discussion}

\subsection{Josephson contact}

In the HPT procedure, the shear stress inserts dislocations and reduces crystallinity, resulting in the grain refinement at the submicrometer or nanometer ranges as shown in Fig. 1. body centered cubic (bcc) body centered tetragonal (bct)

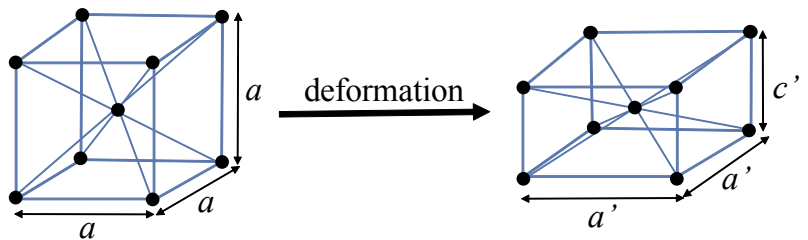

Fig. 17 Schematic of lattice deformation, where a bcc lattice (left panel) is deformed to a bct lattice (right). ${ }^{33)}$

The additional HP compression reduces the intergrain space, and strengthens the intergrain coupling. It has already been confirmed in the experimental side that $T_{\mathrm{c}}$ can increase with strengthening the intergrain coupling. ${ }^{5,6}$ This effect is theoretically suggested to be enhanced in a system with smaller grain size. ${ }^{7}$ ) We assume that the aforementioned effect appears prominently for HPT-Nb $(N=1)$ and all HPTTa $(N=0,2$, and 10) materials.

\subsection{Anisotropic unit-cell compression $\sim$ Change in Density of State by Deforming Lattice}

We have to consider another mechanism for increasing $T_{\mathrm{c}}$ for the HPT-Nb $(N=10)$ materials. According to another work, ${ }^{33)}$ sharp increase in $T_{\mathrm{c}}$ for HPT-Nb $(N=10)$ using the Apiezon-J grease does not appear in the case of good HP condition using MEW and $\mathrm{O}_{2}$. The maximum $T_{\mathrm{c}}$ for HPT-Nb $(N=10)$ using the Apiezon-J oil should be related with the anisotropic contraction of the unit cell. Thus, it is of interest to understand whether or not the anisotropic structure change from bcc to the orthorhombic system close to the tetragonal lattice actually leads to the increase in $T_{\mathrm{c}}$.

We performed density-functional calculations for $\mathrm{Nb}$ using the extended version of the TOKYo AB-INITIO PROgram PACKAGE ${ }^{57)}$ with plane-wave basis sets, where normconserving pseudopotentials ${ }^{58,59)}$ and the generalized gradient approximation of the exchange-correlation potential ${ }^{60)}$ were employed. The cutoff energies in the wavefunction and charge densities were 64 and 256 Ry, respectively, and $40 \times 40 \times 40 k$-point sampling was employed. The electronic density of states (DOS) was calculated by means of the generalized tetrahedron method. ${ }^{61,62)}$

Based on experimental trends in the lattice change, we first focus on the specific deformation of the bcc structure to the bet structure, which is schematically shown in Fig. 17. The deformation is expressed as $(a, a, a) \rightarrow\left(a^{\prime}, a^{\prime}, c^{\prime}\right)$ with $a^{\prime}=$ $(1+\delta) a$ and $c^{\prime}=(1-\delta) a$. In the present calculation, we assume $a=3.29 \AA$ and $\delta=0.015$. The Bravais lattice is expressed for a bcc system as,

$$
\begin{aligned}
& \mathbf{a}_{1}=\frac{a}{2}(-1,1,1), \\
& \mathbf{a}_{2}=\frac{a}{2}(1,-1,1), \\
& \mathbf{a}_{3}=\frac{a}{2}(1,1,-1),
\end{aligned}
$$

and for a bct one as

$$
\mathbf{a}_{1}=\frac{a}{2}[-(1+\delta), 1+\delta, 1-\delta]
$$




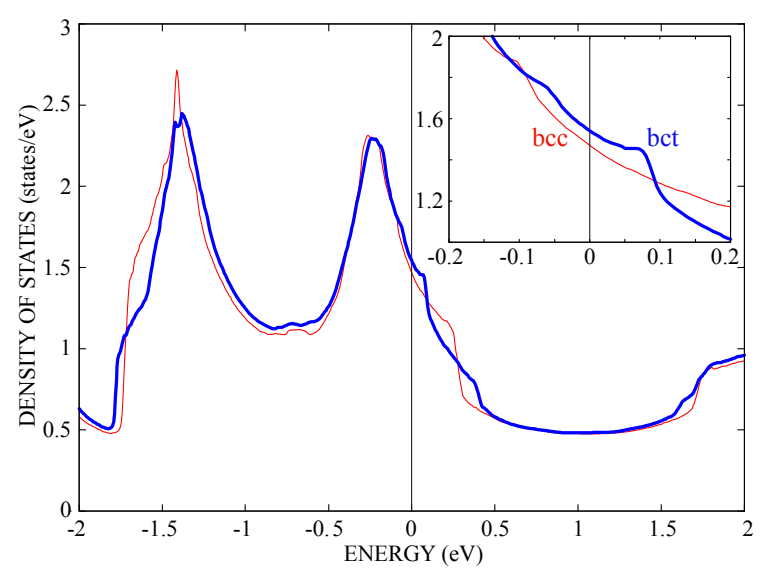

Fig. 18 Calculated electronic density of states of the bcc lattice (thin red curves) and the bct ones (thick blue curves). ${ }^{33)}$ The inset depicts the magnified portion around the Fermi level.

$$
\begin{aligned}
& \mathbf{a}_{2}=\frac{a}{2}[1+\delta,-(1+\delta), 1-\delta], \\
& \mathbf{a}_{3}=\frac{a}{2}[1+\delta, 1+\delta,-(1-\delta)] .
\end{aligned}
$$

Figure 18 depicts the calculated electronic DOS, with the inset depicting the magnification of the low-energy regime. We observe an increase in the DOS at the Fermi level $N(0)$ from the bcc to the bct structures. Here, the value of $N(0)$ is 1.466 (states/eV/atom) for bcc and 1.540 (states/eV/atom) for bct. The order of the present bcc value is reasonably consistent with the past theoretical result. ${ }^{63)}$

By the McMillan-Allen-Dynes formula, $T_{\mathrm{c}}$ can be expressed on a quantitative basis as

$$
T_{\mathrm{c}}=\frac{\omega_{\mathrm{ln}}}{1.2} \exp \left\{-\frac{1.04(1+\lambda)}{\lambda-\mu^{*}(1+0.62 \lambda)}\right\},
$$

where $\omega_{\ln }$ is the phonon frequency, $\lambda$ is the electron lattice coupling, and $\mu^{*}$ is the effective Coulomb potential. ${ }^{64,65)}$ In the case of $\mathrm{Nb}, \lambda$ is of the order of 1 , while $\mu^{*}$ is of the order of $0.1{ }^{66)}$ We can pay much attention to $\lambda$ rather than $\mu^{*}$.

According to McMillan's theoretical work for strongcoupled superconductors, the electron-phonon coupling of the bcc $4 \mathrm{~d}$ transition-metal superconductors is strengthened with the increase in $N(0){ }^{64)}$ An increase in the electronphonon coupling constant as well as that in $N(0)$ contribute to the increase in $T_{\mathrm{c}}$. Consequently, we speculate that the observed $N(0)$ increase due to deformation contributes to the experimental trend of $T_{\mathrm{c}}$ increase.

\subsection{Compression effects on $\mathrm{V}$}

There is no prominent difference in the change in $T_{\mathrm{c}}$ under HP experiments using Ap-J between HPT-V $(N=0)$ and HPT-V $(N=10)$, and they are consistent with the previous data without using PTM by Ishizuka et al. ${ }^{27)}$ The XRD studies by Jenei et al. reported the existence of structural phase transition from bcc to rhombohedral structure around $62 \mathrm{GPa}$ under quasihydrostatic compression in the $\mathrm{Ne}$ pressure medium. ${ }^{67)}$ Under the nonhydrostatic condition, the phase transition occurs at $30 \mathrm{GPa}$ at ambient temperature. Thus, we can assume that the lattice constant monotonically decreases with applying the pressure without the phase transition in the pressure region up to $30 \mathrm{GPa}$. This trend basically leads to the decrease in $N(0)$ and $T_{\mathrm{c}}$. In fact, the experiment exhibits that $T_{\mathrm{c}}$ monotonously increases with an increase in pressure (Fig. 6). Then, the observed increasing trend of $T_{\mathrm{c}}$ in $\mathrm{V}$ is not simply described as the effect of $N(0)$. According to superconducting density functional theory, the superconductivity of $\mathrm{V}$ seems to be beyond the conventional phonon superconductivity; the theoretical $T_{\mathrm{c}}$ based on the phonon mechanism is much overestimated as $16.3 \mathrm{~K}$ compared with the experimental value of $5.4 \mathrm{~K} .{ }^{68)}$ As the possible cause of disagreement, instability of the Cooper pair based on spin fluctuation has been suggested. In general, applying pressure to the system to increase the metallicity would result in suppression of spin fluctuations. Thus, observed positive correlation between the pressure increase and the $T_{\mathrm{c}}$ increase in $\mathrm{V}$ might be explained as suppression of the spin fluctuation with pressure increase. Furthermore, the change in spin fluctuation under pressure may mask the effects of strengthening intergrain-contact and slight structural deformation in the unit-cell level. They are important future problems to be investigated.

\section{Conclusion}

In fifth-group element superconductors $\mathrm{V}, \mathrm{Nb}$, and $\mathrm{Ta}$, artificial material manipulation using the severe plastic deformation and additional hydrostatic compression was attempted to increase the superconducting transition temperature $T_{\mathrm{c}}$. In HPT-Nb with the small $N$ and HPT-Ta for $N=0$, 2 , and 10 , the increase in $T_{\mathrm{c}}$ due to strengthening the intergrain contacts was observed. Further, the prominent increase in $T_{\mathrm{c}}$ for HPT-Nb for $N=10$ originates in the anisotropic deformation of the unit cell. When the quasihydrostatic compression is yielded to the material with heavy residual strain in the unit cell, severe strain accumulated in the bcc unit cell with high symmetry is released at a small amount of additional pressure. In the case of $\mathrm{Nb}$, this manner of lattice deformation is favorable for increasing $T_{\mathrm{c}}$. The knowledge obtained there yields a new strategy to increase $T_{\mathrm{c}}$ from the viewpoints of material science. There would be further potentiality for increasing $T_{\mathrm{c}}$ in the border region between material science using severe plastic deformation and extreme condensed-matter physics using hydrostatic compression.

\section{Acknowledgments}

This work was supported by JSPS KAKENHI Grant Number $17 \mathrm{H} 03379$ and 16K05460. This work was also supported by MEXT KAKENHI, a Grant-in-Aid for Scientific Research (S) (No. 26220909). The HPT process was carried out at the International Research Center on Giant Straining for Advanced Materials (IRC-GSAM) in Kyushu University.

\section{REFERENCES}

1) J.K. Hulm and B.B. Goodman: Phys. Rev. 106 (1957) 659.

2) L.D. Jennings and C.A. Swenson: Phys. Rev. 112 (1958) 31.

3) T.F. Smith: J. Phys. F 2 (1972) 946.

4) M. Strongin: Physica 55 (1971) 155.

5) H. Deguchi, K. Koyama, M. Yasunaka, S. Takagi, N. Nagano and K. 
Mizuno: J. Phys. Chem. Solids 63 (2002) 1081.

6) K. Koyama, H. Deguchi, S. Takagi and K. Mizuno: J. Low Temp. Phys. 131 (2003) 595.

7) J. Mayoh and A.M. García-García: Phys. Rev. B 90 (2014) 134513.

8) R. Valiev, Y. Estrin, Z. Horita, T. Langdon, M. Zehetbauer and Y. Zhu: JOM 58(4) (2006) 33.

9) R. Valiev, R. Islamgaliev and I. Alexandrov: Prog. Mater. Sci. 45 (2000) 103.

10) T. Nishizaki, S. Lee, Z. Horita, T. Sasaki and N. Kobayashi: Physica C 493 (2013) 132.

11) K. Edalati, T. Daio, S. Lee, Z. Horita, T. Nishizaki, T. Akune, T. Nojima and T. Sasaki: Acta Mater. 80 (2014) 149.

12) M. Mito, H. Matsui, K. Tsuruta, T. Yamaguchi, K. Nakamura, H Deguchi, N. Shirakawa, H. Adachi, T. Yamasaki, H. Iwaoka, Y. Ikoma and Z. Horita: Sci. Rep. 6 (2016) 36337.

13) P.W. Bridgman: Phys. Rev. 48 (1935) 825.

14) N.A. Smirnova, V.I. Levit, V.I. Pilyugin, R.I. Kuznetsov, L.S Davydova and V.A. Sazonova: Fiz. Met. Metalloved. 61 (1986) 1170

15) Y. Harai, Y. Ito and Z. Horita: Scr. Mater. 58 (2008) 469.

16) K. Edalati and Z. Horita: Mater. Sci. Eng. A 652 (2016) 325.

17) A. Zhilyaev and T. Langdon: Prog. Mater. Sci. 53 (2008) 893.

18) V.M. Segal, V.I. Reznikov, A.E. Drobyshevskiy and V.I. Kopylov: Russian Metall. 1 (1981) 99.

19) A. Azushima, R. Kopp, A. Korhonen, D.Y. Yang, F. Micari, G.D Lahoti, P. Groche, J. Yanagimoto, N. Tsuji, A. Rosochowski and A. Yanagida: CIRP Ann. Manuf. Tech. 57 (2008) 716.

20) Y. Saito, H. Utsunomiya, N. Tsuji and T. Sakai: Acta Mater. 47 (1999) 579.

21) J.S. Schilling: Handbook of High-Temperature Superconductivity, ed. by J.R. Schrieffer, (Springer, New York, 2007) pp. 427-462.

22) M. Debessai, J.J. Hamlin and J.S. Schilling: Phys. Rev. B 78 (2008) 064519.

23) W.E. Gardner and T.F. Smith: Phys. Rev. 144 (1966) 233.

24) D. Köhnlein: Z. Phys. 208 (1968) 142.

25) N.B. Brabdt and O.A. Zarubia: Sov. Phys. Solid State 15 (1973) 2281

26) Y. Akahama, M. Kobayashi and H. Kawamura: J. Phys. Soc. Jpn. 64 (1995) 4049.

27) M. Ishizuka, M. Iketani and S. Endo: Phys. Rev. B 61 (2000) R3823

28) V.V. Struzhkin, Y.A. Timofeev, R.J. Hemley and H.K. Mao: Phys. Rev. Lett. 79 (1997) 4262

29) W. Gey and G. van Heyden: Z. Phys. 193 (1966) 65

30) W. Gey: Z. Phys. 229 (1969) 85

31) N.B. Brandt and E. Papp: Sov. Phys. JETP 30 (1970) 595.

32) T.F. Smith: Phys. Lett. 33 (1970) 465.

33) M. Mito, Y. Kitamura, T. Tajiri, K. Nakamura, R. Shiraishi, K. Ogata H. Deguchi, T. Yamaguchi, N. Takeshita, T. Nishizaki, K. Edalati and Z. Horita: J. Appl. Phys. 125 (2019) 125901.

34) J. Hatton: Phys. Rev. 103 (1956) 1167.

35) M.D. Fiske: J. Phys. Chem. Solids 2 (1957) 191.

36) S. Lee and Z. Horita: Mater. Trans. 53 (2012) 38

37) K. Edalati and Z. Horita: Acta Mater. 59 (2011) 6831.

38) K. Edalati, J.M. Cubero-Sesin, A. Alhamidi, I. Mohamed and Z. Horita: Mater. Sci. Eng. A 613 (2014) 103

39) P. Das, C.V. Tomy, S.S. Banerjee, H. Takeya, S. Ramakrishnan and A.K. Grover: Phys. Rev. B 78 (2008) 214504.
40) T. Nishizaki: private communication (2019).

41) M. Mito, Y. Komorida, H. Tsuruda, J. Tse, S. Desgreniers, Y. Ohishi, A. Leitch, K. Cvrkalj, C. Robertson and R. Oakley: J. Am. Chem. Soc. 131 (2009) 16012.

42) A.A. Leitch, K. Lekin, S.M. Winter, L.E. Downie, H. Tsuruda, J.S. Tse, M. Mito, S. Desgreniers, P.A. Dube, S. Zhang, Q. Liu, C. Jin, Y. Ohishi and R.T. Oakley: J. Am. Chem. Soc. 133 (2011) 6051.

43) H. Tsuruda, M. Mito, H. Deguchi, S. Takagi, A. Leitch, K. Lekin, S. Winter and R. Oakley: Polyhedron 30 (2011) 2997.

44) S. Yamaguchi, N. Yamaguchi, M. Mito, H. Deguchi, P.J. Baker, S.J. Blundell, M.J. Pitcher, D.R. Parker and S.J. Clarke: J. Korean Phys. Soc. 63 (2013) 445

45) M. Mito, T. Imakyurei, H. Deguchi, K. Matsumoto, H. Hara, T. Ozaki, H. Takeya and Y. Takano: J. Phys. Soc. Jpn. 83 (2014) 023705.

46) M. Mito, H. Matsui, H. Goto, H. Deguchi, K. Matsumoto, H. Hara, T. Ozaki, H. Takeya and Y. Takano: J. Phys. Soc. Jpn. 85 (2016) 024711.

47) M. Mito, K. Ogata, H. Goto, K. Tsuruta, K. Nakamura, H. Deguchi, T. Horide, K. Matsumoto, T. Tajiri, H. Hara, T. Ozaki, H. Takeya and Y. Takano: Phys. Rev. B 95 (2017) 064503.

48) T. Tomita, J.S. Schilling, L. Chen, B.W. Veal and H. Claus: Phys. Rev. B 74 (2006) 064517.

49) M. Mito, M. Hitaka, T. Kawae, K. Takeda, T. Kitai and N. Toyoshima: Jpn. J. Appl. Phys. 40 (2001) 6641.

50) M. Mito: J. Phys. Soc. Jpn. Suppl. A 76 (2007) 182.

51) G.J. Piermarini, S. Block, J.D. Barnett and R.A. Forman: J. Appl. Phys. 46 (1975) 2774

52) M. Ishizuka: Rev. Sci. Instrum. 76 (2005) 123902.

53) M. Ishizuka, K. Amaya and S. Endo: Rev. Sci. Instrum. 66 (1995) 3307.

54) K. Irie, K. Shibayama, M. Mito, S. Takagi, M. Ishizuka, K. Lekin and R.T. Oakley: Phys. Rev. B 99 (2019) 014417.

55) A. Fujiwara, K. Ishii, T. Watanuki, H. Suematsu, H. Nakao, K. Ohwada, Y. Fujii, Y. Murakami, T. Mori, H. Kawada et al.: J. Appl. Cryst. 33 (2000) 1241

56) T. Varga, A.P. Wilkinson and R.J. Angel: Rev. Sci. Instrum. 74 (2003) 4564.

57) J. Yamauchi, M. Tsukada, S. Watanabe and O. Sugino: Phys. Rev. B 54 (1996) 5586

58) N. Troullier and J.L. Martins: Phys. Rev. B 43 (1991) 1993.

59) L. Kleinman and D.M. Bylander: Phys. Rev. Lett. 48 (1982) 1425.

60) J.P. Perdew, K. Burke and M. Ernzerhof: Phys. Rev. Lett. 77 (1996) 3865.

61) T. Fujiwara, S. Yamamoto and Y. Ishii: J. Phys. Soc. Jpn. 72 (2003) 777.

62) Y. Nohara, S. Yamamoto and T. Fujiwara: Phys. Rev. B 79 (2009) 195110.

63) K.H. Lee and K.J. Chang: Phys. Rev. B 54 (1996) 1419.

64) W.L. McMillan: Phys. Rev. 167 (1968) 331.

65) P.B. Allen and R.C. Dynes: Phys. Rev. B 12 (1975) 905.

66) R. Akashi, K. Nakamura, R. Arita and M. Imada: Phys. Rev. B 86 (2012) 054513.

67) Z. Jenei, H.P. Liermann, H. Cynn, J.-H.P. Klepeis, B.J. Baer and W.J. Evans: Phys. Rev. B 83 (2011) 054101.

68) N.N. Lathiotakis, M.A.L. Marques, M. Lüders, L. Fast and E.K.U. Gross: Int. J. Quantum Chem. 99 (2004) 790. 